\title{
Three component Kabachnik-Fields condensation leading to substituted aminomethane- $P$-hydroxymethylphosphonic acids as a tool for screening of bacterial urease inhibitors
}

\author{
Stamatia Vassiliou, ${ }^{\text {A }}$ Annieszka Grabowiecka, ${ }^{\text {Paulina Kosikowska, }}$, \\ and Lukasz Berlicki ${ }^{\mathrm{a}}$ * \\ ${ }^{a}$ Department of Bioorganic Chemistry, Faculty of Chemistry, Wrockaw University of Technology, \\ Wybrzeże Wyspiańskiego 27, 50-370 Wrocław, Poland \\ ${ }^{b}$ Laboratory of Organic Chemistry, Department of Chemistry, University of Athens, \\ Panepistimiopolis, Zografou, 15701 Athens, Greece \\ E-mail: lukasz.berlicki@pwr.wroc.pl
}

Dedicated to Prof. Pawel Kafarski to honor the achievements within his career

\begin{abstract}
Condensation of hydroxyalkane- $H$-phosphinic acids, formaldehyde and secondary amines has given entry to the synthesis of variously substituted aminomethane- $P$-hydroxymethylphosphinic acids. The proposed strategy allowed to perform a feasible synthesis of several molecules with designed biological activity towards bacterial urease - an enzyme which is a medicinally relevant molecular target. The inhibitory potency of compounds was validated using enzyme purified from Bacillus pasteurii.
\end{abstract}

Keywords: Phosphinic acids, multi-component reaction, urea hydrolysis, enzyme inhibitors, Kabachnik-Fields reaction

\section{Introduction}

Multi-component reactions (MCR) have been extensively used for screening compounds directed against a wide variety of molecular targets of medicinal relevance. ${ }^{1-4}$ This approach offers the possibility of producing large number of compounds with the same scaffold and diverse side chains, thus allowing structure optimization in respect of activity, selectivity and other important pharmacological features of the active molecule. Although the most widely studied MCR is Ugitype condensation, other reactions are also used. Among them, Mannich-type three component condensation leading to aminophosphonic acids was applied for construction of compounds with interesting biological activities. ${ }^{5,6}$ 
Urease - the enzyme catalyzing hydrolysis of urea, is a molecular target for potential therapeutics. ${ }^{7,8}$ Ureolytic activity of human pathogens Helicobacter pylori and Proteus mirabilis is the main causative factor of severe clinical conditions concerning the gastric and urinary tract. Ammonia and carbon dioxide released in urea hydrolysis are crucial for $H$. pylori infections pathogenesis. High ammonia concentration results in local $\mathrm{pH}$ increase in stomach. Urease activity may have serious consequences including development of gastric ulcer and/or duodenal ulcers, MALT lymphoma, Ménétrier disease and gastric cancer. ${ }^{9}$ Moreover, infection of the human and animal urinary tract by urease producing species results in urolithiasis. Ammonia elevates urine $\mathrm{pH}$ causing a precipitation of magnesium and/or calcium salts as struvite and carbonate apatite. To confront disadvantages of antibiotic treatment in urease-producing bacterial infections, substances that inhibit urease activity have been intensively studied. ${ }^{10}$ Several classes of urease inhibitors were investigated including urea derivatives, phosphoramidates, hydroxamic acids, quinones, and polyphenols. Amides of phosphoric acid, being classical transition state analogs, represent the group with the highest activity, but unfortunately their utility is strongly limited by low hydrolytic stability (the reported half-life of $N$-(diaminophosphinyl)-4fluorobenzamide, flurofamide at $\mathrm{pH} 2$ is around $5 \mathrm{~min}.){ }^{11}$ Interestingly, a moderately active urease inhibitor - acetohydroxamic acid was approved by FDA in 1983 for urinary tract infections treatment (trademarks Lithostat, Uronefrex), although it exhibits severe side effects including teratogenicity, psychoneurologic and musculo-integumentary symptoms. ${ }^{12,13}$

In this paper we apply a multi-component reaction strategy for screening urease's phosphinate inhibitors. The use of the Kabachnik-Fields three-component condensation of a hydroxyalkane- $H$-phosphinic acid, formaldehyde and a secondary amine to produce substituted aminomethane- $P$-hydroxymethylphosphinic acids is studied. The optimization of a straightforward method for the synthesis of compounds with potential anti-urease activity gives the possibility to assay a variety of analogues.

\section{Results and Discussion}

\section{Design}

Our previous studies have shown that aminophosphinic acids are effective urease inhibitors, in particular those constructed on the basis of aminomethanephosphinic acid fragment. ${ }^{14-16} \mathrm{~N}$ Methylaminomethane- $P$-hydroxymethylphosphinic acid $\mathbf{1}$ was one of the most effective analogues. Using the structure of $\mathbf{1}$ as a scaffold, two types of modifications were envisaged (Scheme 1), namely the substitution of hydroxymethyl fragment (compounds 2-6), or amine moiety (structures 7-12). In order to test the synthetic methodology as well as the complementarity with the enzyme active site, substituents of various size and character (aliphatic/aromatic) were proposed. 

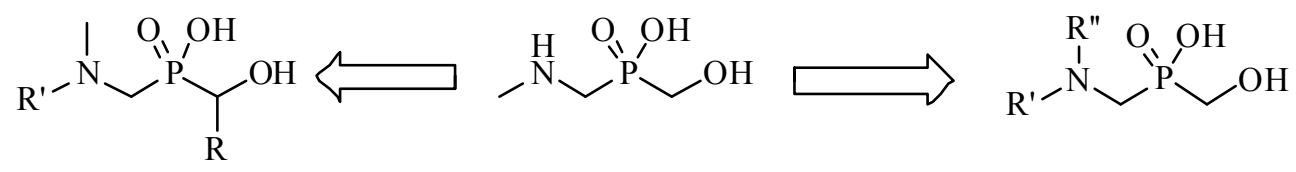

$$
\begin{aligned}
& \text { 2, } R=M e, R^{\prime}=H \\
& \text { 3, R }=M e, R^{\prime}=M e \\
& \text { 4, R }=P h, R^{\prime}=H \\
& \text { 5, R }=P h, R^{\prime}=M e \\
& \text { 6, R }=\text { Bzl, } R^{\prime}=M e
\end{aligned}
$$$$
1
$$

$$
\begin{aligned}
& \text { 7, } R^{\prime}=\text { Et }, R^{\prime \prime}=H \\
& \mathbf{8}, R^{\prime}=\text { Et }, R^{\prime \prime}=\text { Et } \\
& \text { 9, } R^{\prime}=\text { Hex, } R^{\prime \prime}=H \\
& \text { 10, } R^{\prime}=\text { Hex, } R^{\prime \prime}=\text { Me } \\
& \text { 11, } R^{\prime}=\text { c-Hex, } R^{\prime \prime}=H \\
& \text { 12, } R^{\prime}=\text { c-Hex, } R^{\prime \prime}=\text { Me }
\end{aligned}
$$

Scheme 1. The outline of urease inhibitors screening procedure.

\section{Synthesis}

Inhibitors construction started with the preparation of $\alpha$-hydroxyalkanephosphinic acids. Known methods of preparation of these compounds include a variation of the Pudovik addition reaction of commercially available $50 \mathrm{wt} \%$ aqueous hypophosphorous acid to aldehydes under acidic conditions. ${ }^{17}$ Mono- and bis( $\alpha$-hydroxyalkyl)phosphinic acids are obtained with prolonged heating. In a previous paper ${ }^{15}$ we reported the optimized reaction conditions for the synthesis of hydroxymethane- $H$-phosphinic acid 16, which was used in the next step without being isolated (Scheme 2). The same reaction conditions e.g. $50 \mathrm{wt} \%$ aqueous hypophosphorous acid with 1.2 equiv. of aldehyde (acetaldehyde for 13, benzaldehyde for 14, 2-phenylacetaldehyde for 15) in the presence of 1.2 equiv. 6M hydrochloric acid in ethanol refluxed for $48 \mathrm{~h}$, afforded the $\alpha$ hydroxyalkane- $H$-phosphinic acids $\mathbf{1 3 - 1 5}$ as main products $(60-89 \%)$, accompanied by the corresponding bis-adducts. The latter compounds give signals around $40 \mathrm{ppm}$ in ${ }^{31} \mathrm{P}$ NMR while the former around $33 \mathrm{ppm}$. The chemical shift of the carbon atom in the $\alpha$-position of the phosphorus $\left(\delta\left({ }^{13} \mathrm{C}\right) \sim 69 \mathrm{ppm}\right)$ and its coupling constant with the phosphorus atom $\left({ }^{1} \mathrm{~J}_{\mathrm{PC}} \sim 110\right.$ $\mathrm{Hz}$ ) are consistent with the presence of an $\alpha$-hydroxyalkanephosphinic moiety. The $\mathrm{CH}_{\alpha}$ proton resonates in most cases as a multiplet at about $3.6 \mathrm{ppm}$.

Since $\alpha$-hydroxyalkane- $H$-phosphinic acids were obtained, the one pot synthesis of substituted $\alpha_{1}$-aminomethane- $\alpha_{2}$-hydroxyalkanephosphinic acids was available by the classical Kabachnik-Fields three-component reaction. ${ }^{18}$ Several $N, N$-disubstituted phosphinates $\mathbf{3}, \mathbf{5 , 6}, \mathbf{8}$, 10, 12, 17-21 were obtained in around $65 \%$ yield by combining the $\alpha$-hydroxyalkane- $H$ phosphinic acid, formaldehyde, and the secondary amine. When the phosphinate planned to be $\mathrm{N}$-monosubstituted, the $\mathrm{N}$-benzyl substituent was easily removed by catalytic hydrogenolysis after the three-component condensation. Compounds 3, 5, 6, 8, 10, 12, 17-21 were purified by dowex cation exchange resin followed, if necessary, by reversed phase silica gel column chromatography. Most of the secondary amines used were commercially available while the others were synthesized in a stepwise procedure of reductive amination. This involves imine formation in methanol followed by reduction with $\mathrm{NaBH}_{4}$, a procedure which is known to avoid the usual dialkylation problem. ${ }^{19}$ 


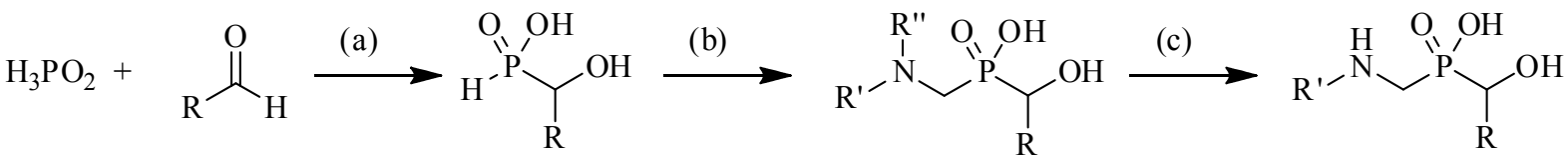

$$
\begin{aligned}
& \text { 13, } \mathrm{R}=\mathrm{Me} \quad 3, \mathrm{R}=\mathrm{R}^{\prime}=\mathrm{R}^{\prime \prime}=\mathrm{Me}, \\
& \text { 14, } \mathrm{R}=\mathrm{Ph} \quad 5, \mathrm{R}=\mathrm{Ph}, \mathrm{R}^{\prime}=\mathrm{R}^{\prime \prime}=\mathrm{Me} \\
& 15, \mathrm{R}=\mathrm{Bzl} \quad 6, \mathrm{R}=\mathrm{Bzl}, \mathrm{R}^{\prime}=\mathrm{R}^{\prime \prime}=\mathrm{Me} \\
& 16, \mathrm{R}=\mathrm{H} \quad 17, \mathrm{R}=\mathrm{R}^{\prime}=\mathrm{Me}, \mathrm{R}^{\prime \prime}=\mathrm{Bzl} \\
& \text { 18, } \mathrm{R}=\mathrm{Ph}, \mathrm{R}^{\prime}=\mathrm{Me}, \mathrm{R}^{\prime \prime}=\mathrm{Bzl} \quad 4, \mathrm{R}=\mathrm{Ph}, \mathrm{R}^{\prime}=\mathrm{Me} \\
& 8, \mathrm{R}=\mathrm{H}, \mathrm{R}^{\prime}=\mathrm{R}^{\prime \prime}=\mathrm{Et} \\
& \text { 10, } R=H, R^{\prime}=M e, R^{\prime \prime}=\text { Hex } \\
& \text { 12, } \mathrm{R}=\mathrm{H}, \mathrm{R}^{\prime}=\mathrm{Me}, \mathrm{R}^{\prime \prime}=\mathrm{c}-\mathrm{Hex} \\
& \text { 19, } \mathrm{R}=\mathrm{H}, \mathrm{R}^{\prime}=\mathrm{Et}, \mathrm{R}^{\prime \prime}=\mathrm{Bzl} \quad \text { 7, } \mathrm{R}=\mathrm{H}, \mathrm{R}^{\prime}=\mathrm{Et} \\
& \text { 20, } \mathrm{R}=\mathrm{H}, \mathrm{R}^{\prime}=\text { Hex, } \mathrm{R}^{\prime \prime}=\mathrm{Bzl} \quad \text { 9, } \mathrm{R}=\mathrm{H}, \mathrm{R}^{\prime}=\text { Hex } \\
& \text { 21, } \mathrm{R}=\mathrm{H}, \mathrm{R}^{\prime}=\mathrm{c}-\mathrm{Hex}, \mathrm{R}^{\prime \prime}=\mathrm{Bzl} \quad \mathbf{1 1}, \mathrm{R}=\mathrm{H}, \mathrm{R}^{\prime}=\mathrm{c}-\mathrm{Hex}
\end{aligned}
$$

Scheme 2. (a) $6 \mathrm{M} \mathrm{HCl}$, ethanol, reflux, $48 \mathrm{~h}$ (b) (i) (R')(R")NH, $6 \mathrm{M} \mathrm{HCl}, 90{ }^{\circ} \mathrm{C}$; (ii) $\mathrm{HCHO}, 90$ ${ }^{\circ} \mathrm{C}, 5 \mathrm{~h} \mathrm{(c)} 10 \% \mathrm{Pd} / \mathrm{C}$, methanol/water, $\mathrm{H}_{2}$.

\begin{tabular}{|c|c|c|c|}
\hline No. & Structure & $\mathrm{IC}_{50}[\mu \mathrm{M}]$ & $K_{\mathrm{i}}[\mu \mathrm{M}]$ \\
\hline 2 & & $1875 \pm 85$ & $487 \pm 9$ \\
\hline 3 & & $1174 \pm 18$ & $352 \pm 26$ \\
\hline 4 & & $943 \pm 17$ & $112 \pm 28$ \\
\hline 5 & & NI & NI \\
\hline
\end{tabular}

\section{Biological activity}

The obtained compounds were tested against urease purified from Bacillus pasteurii (Table 1) using color indophenol assay. Inhibition constants for compounds 2-4, 7, 9, 11 were in micromolar range. The highest activities were exhibited by compounds which were mono $N$ substituted analogues 2, 4, 7, 9 and 11. Within this group $N$-cyclohexyl-aminomethane- $P$ hydroxymethylphosphinic acid (11) was the most potent with $K_{\mathrm{i}}=92 \mu \mathrm{M}$. With the exception of structure 3, $N, N$-disubstituted compounds were found to be inactive. In comparison to the starting structure $\mathbf{1}$, the tested modifications did not lead to improvement of inhibitory activity.

Table 1. Inhibitory activity $\left(\mathrm{IC}_{50}\right.$ and $K_{\mathrm{i}}$ ) of analyzed compounds against Bacillus pasteurii urease 
Table 1. (Continued)

6<smiles>CN(C)CP(=O)(O)C(O)Cc1ccccc1</smiles>

7<smiles>CCNCP(=O)(O)CO</smiles>

8<smiles>CCN(CC)CP(=O)(O)CO</smiles>

9<smiles>CCCCCCNCP(=O)(O)CO</smiles>

10<smiles>CCCCCCN(C)CP(=O)(O)CC</smiles>

12
NI

NI

$270 \pm 70$

NI

$877 \pm 80$

$245 \pm 54$

NI

NI

$520 \pm 49$

$92 \pm 19$

NI
$115 \pm 44$

NI
$\mathrm{NI}$

\section{Conclusions}

The present work describes the application of the three component Kabachnik-Fields reaction for the synthesis of a library of urease inhibitors. The development of a simple synthetic method to obtain compounds with the designed scaffold was achieved. The proposed synthetic approach is convenient for further optimization of aminophosphinic inhibitors of urease.

\section{Experimental Section}

General. Purification of compounds by column chromatography was carried out on silica gel (70-230 mesh), reverse phase silica gel (Kieselgel 60, RP-18, 40-63 $\mu \mathrm{m}$ ), and Dowex AG50 x 4 cation exchange resin. In NMR measurements, $\mathrm{CDCl}_{3}, \mathrm{D}_{2} \mathrm{O}$ and DMSO- $d_{6}$ were used as solvents. ${ }^{1} \mathrm{H},{ }^{31} \mathrm{P}$, and ${ }^{13} \mathrm{C}$ NMR spectra were recorded on a $200 \mathrm{MHz}$ spectrometer. Proton and carbon chemical shifts are referenced to residual solvent. ${ }^{31} \mathrm{P}$ chemical shifts are reported on $\delta$ scale (in ppm) downfield from $85 \% \mathrm{H}_{3} \mathrm{PO}_{4}$. The following abbreviations were used to explain the NMR multiplicities: s ) singlet, d ) doublet, $\mathrm{t}$ ) triplet, q ) quartet, $\mathrm{m}$ ) multiplet, br ) broad. ESI mass 
spectral analyses were performed on a mass spectrometer MSQ Surveyor, Finnigan using direct sample injection. Negative or positive ion ESI spectra were acquired by adjusting the needle and cone voltages accordingly.

\section{General procedure for the synthesis of $\alpha$-hydroxyalkane- $\boldsymbol{H}$-phosphinic acids}

Hypophosphorous acid (50\% aq.) (10 mmol) was added to the solution of the aldehyde (12 $\mathrm{mmol})$ in ethanol $(4 \mathrm{~mL})$ and $6 \mathrm{M}$ aqueous hydrochloric acid $(12 \mathrm{mmol})$. The resulting mixture was refluxed for $48 \mathrm{~h}$. The reaction mixture was concentrated in vacuo and the residue was purified with column chromatography using $\mathrm{CH}_{2} \mathrm{Cl}_{2}-\mathrm{MeOH}-\mathrm{AcOH}$ : 9-1-0.5 as eluent in the case of 13-15 or used without further purification in the case of $\mathbf{1 6}$.

1-Hydroxyethane- $\boldsymbol{H}$-phosphinic acid (13). Yield 87\%; liquid. ${ }^{1} \mathrm{H}$ NMR $\left(200 \mathrm{MHz}, \mathrm{D}_{2} \mathrm{O}\right) \delta$ 1.05-1.17 $\left(2 \times \mathrm{d}, 3 \mathrm{H}, J=7.3 \mathrm{~Hz}, \mathrm{CH}_{3}\right), 3.68(\mathrm{q}, 1 \mathrm{H}, J=7.3 \mathrm{~Hz}, \mathrm{CH}), 6.61\left(\mathrm{~d}, 1 \mathrm{H}, J_{P H}=535.5\right.$ $\mathrm{Hz}, \mathrm{PH}) .{ }^{13} \mathrm{C}$ NMR $\left(50 \mathrm{MHz}, \mathrm{D}_{2} \mathrm{O}\right) \delta 13.8\left(\mathrm{CH}_{3}\right), 63.9\left(\mathrm{~d}, J_{P C}=110.9 \mathrm{~Hz}, \mathrm{CHP}\right) .{ }^{31} \mathrm{P} \mathrm{NMR}(81$ $\left.\mathrm{MHz}, \mathrm{D}_{2} \mathrm{O}\right) \delta 35.1$.

1-Hydroxy-1-phenylmethane- $\boldsymbol{H}$-phosphinic acid (14). Yield 69\%; solid. ${ }^{1} \mathrm{H}$ NMR (200 MHz, $\left.\mathrm{D}_{2} \mathrm{O}\right) \delta 4.72(\mathrm{~d}, 1 \mathrm{H}, J=9.4 \mathrm{~Hz}, \mathrm{CH}), 6.45\left(\mathrm{~d}, 1 \mathrm{H}, J_{P H}=524.2 \mathrm{~Hz}, \mathrm{PH}\right), 7.24\left(\mathrm{~s}, 5 \mathrm{H}\right.$, arom). ${ }^{13} \mathrm{C}$ NMR $\left(50 \mathrm{MHz}, \mathrm{D}_{2} \mathrm{O}\right) \delta 71.6\left(\mathrm{~d}, J_{P C}=113.9 \mathrm{~Hz}, \mathrm{CHP}\right), 126.9,127.0,128.6,128.7,135.0(6 \mathrm{C}$, arom). ${ }^{31} \mathrm{P}$ NMR (81 MHz, $\left.\mathrm{D}_{2} \mathrm{O}\right) \delta 31.6$.

1-Hydroxy-2-phenylethane- $\boldsymbol{H}$-phosphinic acid (15). Yield 60\%; solid. ${ }^{1} \mathrm{H}$ NMR (200 MHz, DMSO) $\delta 2.44(\mathrm{bs}, 1 \mathrm{H}, \mathrm{OH}), 2.61-2.86$ (AB system, $\left.2 \mathrm{H}, J=5.3 \mathrm{~Hz}, \mathrm{CH}_{2} \mathrm{Ph}\right), 3.68(\mathrm{t}, 1 \mathrm{H}, J=3.6$ $\mathrm{Hz}, \mathrm{CHOH}), 6.81\left(\mathrm{~d}, 1 \mathrm{H}, J_{P H}=511.1 \mathrm{~Hz}, \mathrm{PH}\right), 7.26\left(\mathrm{~s}, 5 \mathrm{H}\right.$, arom). ${ }^{13} \mathrm{C}$ NMR $(50 \mathrm{MHz}, \mathrm{DMSO})$ $\delta 36.1\left(\mathrm{CH}_{2} \mathrm{Ph}\right), 70.1\left(\mathrm{~d}, J_{P C}=109.4 \mathrm{~Hz}, \mathrm{CHP}\right), 127.1,128.5,128.8,136.7\left(6 \mathrm{C}\right.$, arom) ${ }^{31} \mathrm{P}$ NMR (81 MHz, DMSO) $\delta 34.3$.

\section{General procedure for the synthesis of secondary amines}

To the solution of the aldehyde $(10 \mathrm{mmol})$ in methanol $(10 \mathrm{~mL})$ the amine $(13 \mathrm{mmol})$ was added. After stirring at room temperature for $1 \mathrm{~h}$, the solution was cooled to $0^{\circ} \mathrm{C}$ prior to adding sodium borohydride $(5 \mathrm{mmol}$ ) portionwise. The resulting solution was stirred at room temperature for 1 $h$ and then evaporated under vacuum. After addition of water and $\mathrm{Et}_{2} \mathrm{O}$, the organic phase was washed with $\mathrm{NaHCO}_{3}$, water, dried over $\mathrm{Na}_{2} \mathrm{SO}_{4}$ and concentrated under vacuum. Column chromatography purification, using EtOAc-MeOH: 9-1 as eluent, provided secondary amines as colorless oils.

N-Benzylmethylamine (22). Yield 60\%; liquid. ${ }^{1} \mathrm{H} \mathrm{NMR}\left(200 \mathrm{MHz}, \mathrm{CDCl}_{3}\right) \delta 1.36(\mathrm{~s}, 1 \mathrm{H}, \mathrm{NH})$, $2.39\left(\mathrm{~s}, 3 \mathrm{H}, \mathrm{CH}_{3}\right), 3.67\left(\mathrm{~s}, 2 \mathrm{H}, \mathrm{CH}_{2}\right), 7.26$ (s, 5H, arom). ${ }^{13} \mathrm{C} \mathrm{NMR}\left(50 \mathrm{MHz}, \mathrm{CDCl}_{3}\right) \delta 36.2$ $\left(\mathrm{CH}_{3}\right), 56.3\left(\mathrm{CH}_{2}\right), 127.1,128.3,128.5,140.5$ (6C, arom).

$\boldsymbol{N}$-Benzylethylamine (23). Yield 70\%; liquid. ${ }^{1} \mathrm{H}$ NMR $\left(200 \mathrm{MHz}, \mathrm{CDCl}_{3}\right) \delta 1.08(\mathrm{t}, 3 \mathrm{H}, J=7.1$ $\mathrm{Hz}, \mathrm{CH}_{3}$ ), 1.41 (s, 1H, NH), 2.60 (q, 2H, J=7.1 Hz, $\mathrm{CH}_{2}$ ), 3.71 (s, 2H, $\mathrm{CH}_{2} \mathrm{Ph}$ ), 7.26 (s, 5H, arom). ${ }^{13} \mathrm{C}$ NMR $\left(50 \mathrm{MHz}, \mathrm{CDCl}_{3}\right) \delta 15.5\left(\mathrm{CH}_{3}\right), 43.8\left(\mathrm{CH}_{2}\right), 54.2\left(\mathrm{CH}_{2} \mathrm{Ph}\right), 127.1,128.3,128.5$, 140.7 (6C, arom). 
$\boldsymbol{N}$-Benzyl-n-hexylamine (24) . Yield 82\%; liquid. ${ }^{1} \mathrm{H}$ NMR $\left(200 \mathrm{MHz}, \mathrm{CDCl}_{3}\right) \delta 0.90(\mathrm{t}, 3 \mathrm{H}, J=$ $\left.7.1 \mathrm{~Hz}, \mathrm{CH}_{3}\right), 1.24-1.52\left(\mathrm{~m}, 8 \mathrm{H},\left(\mathrm{CH}_{2}\right)_{4}\right), 1.97$ (s, 1H, NH), 2.59 (t, 2H, J=7.1 Hz, $\left.\mathrm{CH}_{2}\right), 3.74$ (s, $\left.2 \mathrm{H}, \mathrm{CH}_{2} \mathrm{Ph}\right), 7.24$ (s, 5H, arom). ${ }^{13} \mathrm{C} \mathrm{NMR}\left(50 \mathrm{MHz}, \mathrm{CDCl}_{3}\right) \delta 14.3\left(\mathrm{CH}_{3}\right), 22.9,27.3,30.4$, $32.1,49.8,\left(5 \mathrm{CH}_{2}\right), 54.2\left(\mathrm{CH}_{2} \mathrm{Ph}\right), 127.1,128.3,128.5,140.7$ (6C, arom).

$\mathrm{N}$-Benzylcyclohexylamine (25). Yield 74\%; liquid. ${ }^{1} \mathrm{H} \mathrm{NMR}\left(200 \mathrm{MHz}, \mathrm{CDCl}_{3}\right) \delta$ 0.93-1.25 (m, $\left.7 \mathrm{H},\left(\mathrm{CH}_{2}\right)_{3}+\mathrm{NH}\right), 1.47-1.77\left(\mathrm{~m}, 4 \mathrm{H},\left(\mathrm{CH}_{2}\right)_{2}\right), 2.32(\mathrm{bq}, 1 \mathrm{H}, \mathrm{CH}), 3.61\left(\mathrm{~s}, 2 \mathrm{H}, \mathrm{CH}_{2} \mathrm{Ph}\right), 7.20(\mathrm{~s}$, $5 \mathrm{H}$, arom). ${ }^{13} \mathrm{C}$ NMR (50 MHz, $\left.\mathrm{CDCl}_{3}\right) \delta 25.1\left(2 \mathrm{C}, \mathrm{CH}_{2}\right), 26.4\left(\mathrm{CH}_{2}\right), 33.7\left(2 \mathrm{C}, \mathrm{CH}_{2}\right), 51.2$ $\left(\mathrm{CH}_{2} \mathrm{Ph}\right), 56.3(\mathrm{CH}), 126.8,128.1,128.4,141.2(6 \mathrm{C}$, arom).

\section{General procedure for the synthesis of $\alpha_{1}$-aminoalkane- $\alpha_{2}$-hydroxyalkanephosphinates}

1-Hydroxyalkane- $H$-phosphinic acid $(1 \mathrm{mmol})$ was dissolved in water $(2 \mathrm{~mL})$, followed by the addition of the corresponding secondary amine $(1 \mathrm{mmol})$ and $6 \mathrm{~N} \mathrm{HCl}(0.5 \mathrm{~mL})$. The solution was warmed to $90{ }^{\circ} \mathrm{C}$, $p$-formaldehyde $(2 \mathrm{mmol})$ was added, and the mixture was kept at $90{ }^{\circ} \mathrm{C}$ for additional $5 \mathrm{~h}$. After removing the volatiles, the crude product was dissolved in water and purified using Dowex AG50x4 cation exchange resin with water as eluent. Fractions that gave slightly positive color reaction with ninhydrin and appropriate NMR spectral data were combined and evaporated under vacuum to give the phosphinates as hygroscopic solids. In certain cases an extra purification step was added using reverse phase silica gel and watermethanol (9.5-0.5) as eluent.

$\boldsymbol{N}, \boldsymbol{N}$-Dimethylaminomethane(P-1-hydroxyethyl)phosphinic acid (3). Yield $77 \%$. ${ }^{1} \mathrm{H}$ NMR $\left(200 \mathrm{MHz}, \mathrm{D}_{2} \mathrm{O}\right) \delta$ 0.93-1.05 $\left(2 \times \mathrm{d}, 3 \mathrm{H}, J=7.4 \mathrm{~Hz}, \mathrm{CH}_{3}\right), 2.64\left(\mathrm{~s}, 6 \mathrm{H},\left(\mathrm{CH}_{3}\right)_{2}\right), 3.07(\mathrm{~d}, J=8.6$ $\left.\mathrm{Hz} 2 \mathrm{H}, \mathrm{CH}_{2} \mathrm{P}\right), 3.57(\mathrm{q}, 1 \mathrm{H}, J=7.3 \mathrm{~Hz} \mathrm{CH}) .{ }^{13} \mathrm{C} \mathrm{NMR}\left(50 \mathrm{MHz}, \mathrm{D}_{2} \mathrm{O}\right) \delta 15.2\left(\mathrm{CH}_{3}\right), 45.7$ $\left(\left(\mathrm{CH}_{3}\right)_{2}\right), 54.4\left(\mathrm{~d}, J_{P C}=82.1 \mathrm{~Hz}, \mathrm{CH}_{2} \mathrm{P}\right), 66.4\left(\mathrm{~d}, J_{P C}=122.4 \mathrm{~Hz}, \mathrm{CHP}\right) .{ }^{31} \mathrm{P} \mathrm{NMR}(81 \mathrm{MHz}$, $\left.\mathrm{D}_{2} \mathrm{O}\right) \delta$ 30.5. ES-MS $m / z:(\mathrm{M}-\mathrm{H})=166.2$. Anal. Calcd for $\mathrm{C}_{5} \mathrm{H}_{14} \mathrm{NO}_{3} \mathrm{P}$ (167.07): C, 35.93; $\mathrm{H}$, 8.44; N, 8.38\%. Found: C, 35.76; H, 8.30; N, 8.31\%.

$\boldsymbol{N}, \boldsymbol{N}$-Dimethylaminomethane( $\boldsymbol{P}$-1-hydroxyphenylmethyl)phosphinic acid (5). Yield 79\%. ${ }^{1} \mathrm{H}$ NMR $\left(200 \mathrm{MHz}, \mathrm{D}_{2} \mathrm{O}\right) \delta 2.73\left(\mathrm{~s}, 6 \mathrm{H},\left(\mathrm{CH}_{3}\right)_{2}\right), 3.07(\mathrm{~d}, 2 \mathrm{H}, J=8.7 \mathrm{~Hz} \mathrm{CH})_{2}, 4.67(\mathrm{~d}, 1 \mathrm{H}, J=9.4$ $\mathrm{Hz}, \mathrm{CH}), 7.29\left(\mathrm{~s}, 5 \mathrm{H}\right.$, arom). ${ }^{13} \mathrm{C} \mathrm{NMR}\left(50 \mathrm{MHz}, \mathrm{D}_{2} \mathrm{O}\right) \delta 45.8\left(\left(\mathrm{CH}_{3}\right)_{2}\right), 54.8\left(\mathrm{~d}, J_{P C}=83.2 \mathrm{~Hz}\right.$, $\left.\mathrm{CH}_{2} \mathrm{P}\right), 73.6\left(\mathrm{~d}, J_{P C}=119.9 \mathrm{~Hz}, \mathrm{CHP}\right), 126.9,128.0,128.5,129.0,137.1$ (6C, arom). ${ }^{31} \mathrm{P}$ NMR $\left(81 \mathrm{MHz}, \mathrm{D}_{2} \mathrm{O}\right) \delta$ 26.4. ES-MS $m / z:(\mathrm{M}-\mathrm{H})=228.3$. Anal. Calcd for $\mathrm{C}_{10} \mathrm{H}_{16} \mathrm{NO}_{3} \mathrm{P}$ (229.09): C, 52.40; H, 7.04; N, 6.11\%. Found: C, 52.56; H, 76.93; N, 6.02\%.

$\boldsymbol{N}, \boldsymbol{N}$-Dimethylaminomethane( $\boldsymbol{P}$-1-hydroxy-2-phenylethyl)phosphinic acid (6). Yield $81 \% .{ }^{1} \mathrm{H}$ NMR $\left(200 \mathrm{MHz}, \mathrm{D}_{2} \mathrm{O}\right) \delta 2.69\left(\mathrm{~s}, 6 \mathrm{H},\left(\left(\mathrm{CH}_{3}\right)_{2}\right), 2.75-2.96\right.$ (AB system, $\left.2 \mathrm{H}, J=5.3 \mathrm{~Hz} \mathrm{CH}{ }_{2} \mathrm{Ph}\right)$, $3.01\left(2 \mathrm{H}, \mathrm{d}, J=8.5 \mathrm{~Hz}, \mathrm{CH}_{2} \mathrm{P}\right) 3.62$ (two t, $\left.1 \mathrm{H}, J=3.6 \mathrm{~Hz}, \mathrm{CHOH}\right), 7.09$ (s, $5 \mathrm{H}$, arom). ${ }^{13} \mathrm{C}$ NMR $\left(50 \mathrm{MHz}, \mathrm{D}_{2} \mathrm{O}\right) \delta 38.2\left(\mathrm{CH}_{2} \mathrm{Ph}\right), 45.7\left(\left(\mathrm{CH}_{3}\right)_{2}\right), 54.2\left(\mathrm{~d}, J_{P C}=84.0 \mathrm{~Hz}, \mathrm{CH}_{2} \mathrm{P}\right), 71.2\left(\mathrm{~d}, J_{P C}\right.$ $=120.6 \mathrm{~Hz}, \mathrm{CHP}), 126.8,128.7,129.4,137.6$ (6C, arom). ${ }^{31} \mathrm{P}$ NMR $\left(81 \mathrm{MHz}, \mathrm{D}_{2} \mathrm{O}\right) \delta 28.7$. ESMS $m / z:(M-H)=242.3$ Anal. Calcd for $\mathrm{C}_{11} \mathrm{H}_{18} \mathrm{NO}_{3} \mathrm{P}$ (243.10): C, 54.32; H, 7.46; N, 5.76\%. Found: C, 54.49; H, 7.32; N, 5.64\%.

$\boldsymbol{N}$-Benzyl- $\boldsymbol{N}$-methylaminomethane(P-1-hydroxyethyl)phosphinic acid (17). Yield $66 \%$. ${ }^{1} \mathrm{H}$ NMR $\left(200 \mathrm{MHz}, \mathrm{D}_{2} \mathrm{O}\right) \delta$ 1.05-1.17 $\left(2 \times \mathrm{d}, 3 \mathrm{H}, J=7.4 \mathrm{~Hz} \mathrm{CH}_{3}\right), 2.64\left(\mathrm{~s}, 3 \mathrm{H}, \mathrm{CH}_{3} \mathrm{~N}\right), 2.93(\mathrm{~d}, 2 \mathrm{H}$, 
$J=8.7 \mathrm{~Hz}, \mathrm{CH}_{2}$ ), 3.65 (q, $\left.1 \mathrm{H}, J=7.3 \mathrm{~Hz}, \mathrm{CH}\right), 4.01$ (s, 2H, $\left.\mathrm{CH}_{2} \mathrm{Ph}\right), 7.28$ (s, 5H, arom). ${ }^{13} \mathrm{C}$ NMR $\left(50 \mathrm{MHz}, \mathrm{D}_{2} \mathrm{O}\right) \delta 15.3\left(\mathrm{CH}_{3}\right), 42.9\left(\mathrm{CH}_{3} \mathrm{~N}\right), 52.5\left(\mathrm{~d}, J_{P C}=90.1 \mathrm{~Hz}, \mathrm{CH}_{2} \mathrm{P}\right), 62.6\left(\mathrm{CH}_{2} \mathrm{Ph}\right)$, $67.0\left(\mathrm{~d}, J_{P C}=115.6 \mathrm{~Hz}, \mathrm{CHP}\right), 129.2,129.3,130.7,132.9\left(6 \mathrm{C}\right.$, arom). ${ }^{31} \mathrm{P} \mathrm{NMR}\left(81 \mathrm{MHz}, \mathrm{D}_{2} \mathrm{O}\right)$ $\delta$ 31.7. ES-MS $m / z$ : $(\mathrm{M}+\mathrm{H})=244.0$.

$\boldsymbol{N}$-Benzyl- $\boldsymbol{N}$-methylaminomethane(P-1-hydroxyphenylmethyl)phosphinic acid (18). Yield $57 \%$. ${ }^{1} \mathrm{H}$ NMR $\left(200 \mathrm{MHz}, \mathrm{D}_{2} \mathrm{O}\right) \delta 2.83\left(\mathrm{~s}, 3 \mathrm{H}, \mathrm{CH}_{3}\right), 3.00\left(\mathrm{~d}, 2 \mathrm{H}, J=8.7 \mathrm{~Hz}, \mathrm{CH}_{2}\right) 4.21(\mathrm{~s}, 2 \mathrm{H}$, $\left.\mathrm{CH}_{2} \mathrm{Ph}\right), 4.67(\mathrm{~d}, 1 \mathrm{H}, J=9.4 \mathrm{~Hz}, \mathrm{CH}), 7.24-7.39(\mathrm{~m}, 10 \mathrm{H}$, arom $) .{ }^{13} \mathrm{C}$ NMR $\left(50 \mathrm{MHz}, \mathrm{D}_{2} \mathrm{O}\right) \delta$ $43.0\left(\mathrm{CH}_{3}\right), 51.6\left(\mathrm{~d}, J_{P C}=83.9 \mathrm{~Hz}, \mathrm{CH}_{2} \mathrm{P}\right), 62.6\left(\mathrm{CH}_{2} \mathrm{Ph}\right), 74.2\left(\mathrm{~d}, J_{P C}=114.9 \mathrm{~Hz}, \mathrm{CHP}\right), 126.8$, $127.4,128.0,128.6,128.7,129.4,130.3,131.2,137.4$ (12C, arom). ${ }^{31} \mathrm{P}$ NMR $\left(81 \mathrm{MHz}, \mathrm{D}_{2} \mathrm{O}\right) \delta$ 25.5. ES-MS $m / z:(\mathrm{M}+\mathrm{H})=306.2$.

$\boldsymbol{N}, \boldsymbol{N}$-Diethylaminomethane( $\boldsymbol{P}$-hydroxymethyl)phosphinic acid (8). Yield $41 \% .{ }^{1} \mathrm{H}$ NMR (200 $\left.\mathrm{MHz}, \mathrm{D}_{2} \mathrm{O}\right) \delta 1.15\left(\mathrm{t}, 6 \mathrm{H}, J=7.1 \mathrm{~Hz}, 2 \times \mathrm{CH}_{3}\right), 3.06-3.27\left(\mathrm{~m}, 6 \mathrm{H}, \mathrm{CH}_{2} \mathrm{P}+2 \times \mathrm{CH}_{2} \mathrm{~N}\right), 3.50(\mathrm{~d}$, $\left.2 \mathrm{H}, J=6.9 \mathrm{~Hz}, \mathrm{CH}_{2} \mathrm{OH}\right) .{ }^{13} \mathrm{C} \mathrm{NMR}\left(50 \mathrm{MHz}, \mathrm{D}_{2} \mathrm{O}\right) \delta 8.3\left(\mathrm{CH}_{3}\right), 48.8\left(\mathrm{~d}, J_{P C}=81.7 \mathrm{~Hz}, \mathrm{CH}_{2} \mathrm{P}\right)$, $50.2\left(\mathrm{CH}_{2} \mathrm{CH}_{3}\right), 60.1$ (d, $\left.J_{P C}=117.5 \mathrm{~Hz}, \mathrm{CHP}\right) .{ }^{31} \mathrm{P}$ NMR $\left(81 \mathrm{MHz}, \mathrm{D}_{2} \mathrm{O}\right) \delta 27.6$. ES-MS $m / z$ : $(\mathrm{M}+\mathrm{H})=$ 182.2. Anal. Calcd for $\mathrm{C}_{6} \mathrm{H}_{16} \mathrm{NO}_{3} \mathrm{P}$ (181.09): C, 39.78; H, 8.90; N, 7.73\%. Found: C, $39.89 ; \mathrm{H}, 8.73 ; \mathrm{N}, 7.67 \%$.

$\boldsymbol{N}$-Hexyl- $\boldsymbol{N}$-methylaminomethane(P-hydroxymethyl)phosphinic acid (10). Yield $68 \%$. ${ }^{1} \mathrm{H}$ NMR (200 MHz, $\left.\mathrm{D}_{2} \mathrm{O}\right) \delta 0.64\left(\mathrm{t}, 3 \mathrm{H}, J=7.1 \mathrm{~Hz} \mathrm{CH}_{3}\right), 1.04-1.25\left(\mathrm{~m}, 6 \mathrm{H}, 3\left(\mathrm{CH}_{2}\right)\right), 1.55$ (bt, 2H, $\left.\mathrm{CH}_{2} \mathrm{CH}_{2} \mathrm{~N}\right), 2.78\left(\mathrm{~s}, 3 \mathrm{H}, \mathrm{CH}_{3} \mathrm{~N}\right), 2.90-3.40\left(\mathrm{~m}, 4 \mathrm{H}, \mathrm{CH}_{2} \mathrm{~N}+\mathrm{CH}_{2} \mathrm{P}\right), 3.49(\mathrm{~d}, 2 \mathrm{H}, J=6.3 \mathrm{~Hz}$, $\left.\mathrm{CH}_{2} \mathrm{OH}\right) .{ }^{13} \mathrm{C}$ NMR $\left(50 \mathrm{MHz}, \mathrm{D}_{2} \mathrm{O}\right) \delta 13.3\left(\mathrm{CH}_{3}\right), 21.8\left(\mathrm{CH}_{2}\right), 23.6\left(\mathrm{CH}_{2}\right), 25.2\left(\mathrm{CH}_{2}\right), 30.5$ $\left(\mathrm{CH}_{2}\right), 48.7\left(\mathrm{CH}_{3}\right), 52.7\left(\mathrm{~d}, J_{P C}=84.1 \mathrm{~Hz}, \mathrm{CH}_{2} \mathrm{P}\right), 58.9\left(\mathrm{CH}_{2} \mathrm{~N}\right), 60.2\left(\mathrm{~d}, J_{P C}=122.8 \mathrm{~Hz}, \mathrm{CHP}\right)$, 129.2, 129.4, 130.3, 131.2, 141.2 (6C, arom). ${ }^{31} \mathrm{P}$ NMR $\left(81 \mathrm{MHz}, \mathrm{D}_{2} \mathrm{O}\right) \delta 27.2$. ES-MS $m / z$ : (M$\mathrm{H})=$ 222.3. Anal. Calcd for $\mathrm{C}_{9} \mathrm{H}_{22} \mathrm{NO}_{3} \mathrm{P}$ (223.13): C, 48.42; H, 9.93; N, 6.27\%. Found: C, 48.31; H, 10.06; N, 6.34\%.

$\boldsymbol{N}$-Cyclohexyl- $\boldsymbol{N}$-methylaminomethane( $P$-hydroxymethyl)phosphinic acid (12). Yield 55\%. ${ }^{1} \mathrm{H}$ NMR $\left(200 \mathrm{MHz}, \mathrm{D}_{2} \mathrm{O}\right) \delta 0.80-1.90\left(\mathrm{~m}, 10 \mathrm{H}, 5 \times \mathrm{CH}_{2}\right), 2.76\left(\mathrm{~s}, 3 \mathrm{H}, \mathrm{CH}_{3} \mathrm{~N}\right), 2.90-3.40(\mathrm{~m}, 3 \mathrm{H}$, $\left.\mathrm{CHN}+\mathrm{CH}_{2} \mathrm{P}\right), 3.53\left(\mathrm{~d}, 2 \mathrm{H}, J=6.7 \mathrm{~Hz}, \mathrm{CH}_{2} \mathrm{OH}\right) .{ }^{13} \mathrm{C} \mathrm{NMR}\left(50 \mathrm{MHz}, \mathrm{D}_{2} \mathrm{O}\right) \delta 24.42\left(\mathrm{CH}_{2}\right), 26.1$ 2( $\left(\mathrm{CH}_{2}\right), 39.4\left(\mathrm{CH}_{3}\right), 49.4\left(\mathrm{~d}, J_{P C}=81.3 \mathrm{~Hz}, \mathrm{CH}_{2} \mathrm{P}\right), 60.1$ (d, $\left.J_{P C}=119.0 \mathrm{~Hz}, \mathrm{CH}_{2} \mathrm{P}\right), 67.3(\mathrm{CH})$. ${ }^{31} \mathrm{P}$ NMR $\left(81 \mathrm{MHz}, \mathrm{D}_{2} \mathrm{O}\right) \delta$ 27.7. ES-MS $m / z$ : $(\mathrm{M}-\mathrm{H})=220.3$. Anal. Calcd for $\mathrm{C}_{9} \mathrm{H}_{20} \mathrm{NO}_{3} \mathrm{P}$ (221.12): C, 48.86; H, 9.11; N, 6.33\%. Found: C, 48.68; H, 9.27; N, 6.29\%.

$\boldsymbol{N}$-Benzyl- $\boldsymbol{N}$-ethylaminomethane(P-hydroxymethyl)phosphinic acid (19). Yield $65 \%$. ${ }^{1} \mathrm{H}$ NMR (200 MHz, D $2 \mathrm{O}) \delta 1.15\left(\mathrm{t}, 3 \mathrm{H}, J=7.1 \mathrm{~Hz} \mathrm{CH}_{3}\right), 3.01\left(\mathrm{~m}, 4 \mathrm{H}, \mathrm{CH}_{2} \mathrm{P}+\mathrm{CH}_{2} \mathrm{CH}_{3}\right), 3.45$ (d, $\left.2 \mathrm{H}, J=6.8 \mathrm{~Hz}, \mathrm{CH}_{2} \mathrm{OH}\right), 4.29\left(\mathrm{~s}, 2 \mathrm{H}, \mathrm{CH}_{2} \mathrm{Ph}\right), 7.33\left(\mathrm{~s}, 5 \mathrm{H}\right.$, arom). ${ }^{13} \mathrm{C}$ NMR $\left(50 \mathrm{MHz}, \mathrm{D}_{2} \mathrm{O}\right) \delta$ $8.4\left(\mathrm{CH}_{3}\right), 48.5\left(\mathrm{~d}, J_{P C}=82.4 \mathrm{~Hz}, \mathrm{CH}_{2} \mathrm{P}\right), 50.3\left(\mathrm{CH}_{2} \mathrm{CH}_{3}\right), 60.3\left(\mathrm{~d}, J_{P C}=118.0 \mathrm{~Hz}, \mathrm{CHP}\right) .{ }^{31} \mathrm{P}$ $\mathrm{NMR}\left(81 \mathrm{MHz}, \mathrm{D}_{2} \mathrm{O}\right) \delta$ 27.6. ES-MS $m / z$ : $(\mathrm{M}-\mathrm{H})=242.3$.

$\boldsymbol{N}$-Benzyl- $\boldsymbol{N}$-hexylaminomethane(P-hydroxymethyl)phosphinic acid (20). Yield $61 \%$. ${ }^{1} \mathrm{H}$ NMR $\left(200 \mathrm{MHz}, \mathrm{D}_{2} \mathrm{O}\right) \delta 0.65\left(\mathrm{t}, 3 \mathrm{H}, J=7.1 \mathrm{~Hz} \mathrm{CH}_{3}\right), 1.05-1.21\left(\mathrm{~m}, 6 \mathrm{H}, 3 \times \mathrm{CH}_{2}\right), 1.57$ (bt, 2H, $\mathrm{CH}_{2} \mathrm{CH}_{2} \mathrm{~N}$ ), 3.04 (t, 2H, J=7.9 Hz, $\left.\mathrm{CH}_{2} \mathrm{~N}\right), 3.17$ (d, 2H, J=8.4 Hz, $\left.\mathrm{CH}_{2} \mathrm{P}\right), 3.49$ (d, $2 \mathrm{H}, J=6.2$ $\left.\mathrm{Hz}, \mathrm{CH}_{2} \mathrm{OH}\right), 4.35\left(\mathrm{~s}, 2 \mathrm{H}, \mathrm{CH}_{2} \mathrm{Ph}\right), 7.35$ (s, 5H, arom). ${ }^{13} \mathrm{C} \mathrm{NMR}\left(50 \mathrm{MHz}, \mathrm{D}_{2} \mathrm{O}\right) \delta 13.3\left(\mathrm{CH}_{3}\right)$, $21.8\left(\mathrm{CH}_{2}\right), 23.1\left(\mathrm{CH}_{2}\right), 25.3\left(\mathrm{CH}_{2}\right), 30.4\left(\mathrm{CH}_{2}\right), 48.6\left(\mathrm{CH}_{2} \mathrm{~N}\right), 49.6\left(\mathrm{~d}, J_{P C}=84.8 \mathrm{~Hz}, \mathrm{CH}_{2} \mathrm{P}\right)$, 
$55.0\left(\mathrm{CH}_{2} \mathrm{Ph}\right), 60.4\left(\mathrm{~d}, J_{P C}=125.8 \mathrm{~Hz}, \mathrm{CHP}\right), 129.2,129.4,130.3,131.2,141.2(6 \mathrm{C}$, arom $) .{ }^{31} \mathrm{P}$ NMR $\left(81 \mathrm{MHz}, \mathrm{D}_{2} \mathrm{O}\right) \delta 27.1$. ES-MS $m / z:(\mathrm{M}+\mathrm{H})=300.1$.

$\boldsymbol{N}$-Benzyl- $\boldsymbol{N}$-cyclohexylaminomethane( $\boldsymbol{P}$-hydroxymethyl)phosphinic acid (21). Yield $77 \%$. ${ }^{1} \mathrm{H}$ NMR (200 MHz, $\left.\mathrm{D}_{2} \mathrm{O}\right) \delta 1.00-1.29\left(\mathrm{~m}, 8 \mathrm{H}, 3 \times \mathrm{CH}_{2}\right), 1.40-1.97\left(\mathrm{~m}, 4 \mathrm{H}, 2 \times \mathrm{CH}_{2}\right), 2.01(\mathrm{~m}$, $1 \mathrm{H}, \mathrm{CHN}), 2.96-3.08\left(\mathrm{~m}, 3 \mathrm{H}, \mathrm{CHN}+\mathrm{CH}_{2} \mathrm{P}\right), 3.59\left(\mathrm{~d}, 2 \mathrm{H}, J=6.9 \mathrm{~Hz}, \mathrm{CH}_{2} \mathrm{OH}\right), 3.63(\mathrm{~s}, 2 \mathrm{H}$, $\left.\mathrm{CH}_{2} \mathrm{Ph}\right), 7.35$ (s, 5H, arom). ${ }^{13} \mathrm{C}$ NMR $\left(50 \mathrm{MHz}, \mathrm{D}_{2} \mathrm{O}\right) \delta 24.62\left(\mathrm{CH}_{2}\right), 25.3\left(\mathrm{CH}_{2}\right), 27.12\left(\mathrm{CH}_{2}\right)$, $46.6\left(\mathrm{~d}, J_{P C}=86.8 \mathrm{~Hz}, \mathrm{CH}_{2} \mathrm{P}\right), 56.7\left(\mathrm{CH}_{2} \mathrm{Ph}\right), 60.4\left(\mathrm{~d}, J_{P C}=122.0 \mathrm{~Hz}, \mathrm{CHP}\right), 64.0(\mathrm{CH}), 129.6$, 129.8, 130.3, 131.0, 141.4 (6C, arom). ${ }^{31} \mathrm{P}$ NMR $\left(81 \mathrm{MHz}, \mathrm{D}_{2} \mathrm{O}\right) \delta 27.4$. ES-MS $m / z:(\mathrm{M}+\mathrm{H})=$ 298.0.

\section{General procedure for catalytic hydrogenolysis}

A mixture of $10 \% \mathrm{Pd} / \mathrm{C}(10 \% \mathrm{wt})$ and $\mathrm{N}$-benzylaminoalkanephosphinic acid $(1.50 \mathrm{mmol})$ in methanol-water: 4-1 (10 mL) was stirred at room temperature under atmosphere of hydrogen until all the starting material was consumed as observed by TLC $(2.5 \mathrm{~h})$. The catalyst was filtered off through celite, and the mixture was washed with methanol and water/methanol (1:4). Solvents were combined and evaporated in vacuo to give a $N$-monosubstituted phosphinates that were dried over $\mathrm{P}_{2} \mathrm{O}_{5}$.

$\boldsymbol{N}$-Methylaminomethane(P-1-hydroxyphenylmethyl)phosphinic acid (4). Yield $97 \%$. ${ }^{1} \mathrm{H}$ NMR $\left(200 \mathrm{MHz}, \mathrm{D}_{2} \mathrm{O}\right) \delta 2.60\left(\mathrm{~s}, 3 \mathrm{H}, \mathrm{CH}_{3}\right), 3.02\left(\mathrm{~d}, 2 \mathrm{H}, J=9.8 \mathrm{~Hz}, \mathrm{CH}_{2}\right), 4.67(\mathrm{~d}, 1 \mathrm{H}, J=9.4$ $\mathrm{Hz}, \mathrm{CH}), 7.22\left(\mathrm{~s}, 5 \mathrm{H}\right.$, arom). ${ }^{13} \mathrm{C}$ NMR $\left(50 \mathrm{MHz}, \mathrm{D}_{2} \mathrm{O}\right) \delta 35.4\left(\mathrm{CH}_{3}\right), 46.2\left(\mathrm{~d}, J_{P C}=86.8 \mathrm{~Hz}\right.$, $\left.\mathrm{CH}_{2} \mathrm{P}\right), 73.6\left(\mathrm{~d}, J_{P C}=115.9 \mathrm{~Hz}, \mathrm{CHP}\right), 126.8,126.9,128.0,128.6,137.1$ (6C, arom). ${ }^{31} \mathrm{P}$ NMR $\left(81 \mathrm{MHz}, \mathrm{D}_{2} \mathrm{O}\right) \delta$ 25.8. ES-MS $m / z:(\mathrm{M}+\mathrm{H})=216.1$. Anal. Calcd for $\mathrm{C}_{9} \mathrm{H}_{14} \mathrm{NO}_{3} \mathrm{P}$ (215.07): C, 50.23; H, 6.56; N, 6.51\%. Found: C, 50.43; H, 6.35; N, 6.44\%.

$\boldsymbol{N}$-Methylaminomethane(P-1-hydroxyethyl)phosphinic acid (2). Yield 98\%. ${ }^{1} \mathrm{H}$ NMR (200 $\left.\mathrm{MHz}, \mathrm{D}_{2} \mathrm{O}\right) \delta 1.02-1.17\left(2 \times \mathrm{d}, 3 \mathrm{H}, J=7.4 \mathrm{~Hz}, \mathrm{CH}_{3}\right), 2.58\left(\mathrm{~s}, 3 \mathrm{H}, \mathrm{CH}_{3} \mathrm{~N}\right), 2.98(\mathrm{~d}, 2 \mathrm{H}, J=9.8$ $\left.\mathrm{Hz}, \mathrm{CH}_{2}\right), 3.70(\mathrm{q}, 1 \mathrm{H}, J=7.2 \mathrm{~Hz}, \mathrm{CH}) .{ }^{13} \mathrm{C}$ NMR $\left(50 \mathrm{MHz}, \mathrm{D}_{2} \mathrm{O}\right) \delta 15.3\left(\mathrm{CH}_{3}\right), 35.4\left(\mathrm{CH}_{3} \mathrm{~N}\right)$, $45.9\left(\mathrm{~d}, J_{P C}=86.4 \mathrm{~Hz}, \mathrm{CH}_{2} \mathrm{P}\right), 66.5\left(\mathrm{~d}, J_{P C}=121.0 \mathrm{~Hz}, \mathrm{CHP}\right) .{ }^{31} \mathrm{P}$ NMR $\left(81 \mathrm{MHz}, \mathrm{D}_{2} \mathrm{O}\right) \delta 30.1$. ES-MS $m / z:(\mathrm{M}+\mathrm{H})=154.1$. Anal. Calcd for $\mathrm{C}_{4} \mathrm{H}_{12} \mathrm{NO}_{3} \mathrm{P}$ (153.06): C, 31.38; H, 7.90; N, 9.15\%. Found: C, 31.47; H, 7.85; N, 9.20\%.

$\boldsymbol{N}$-Ethylaminomethane(P-hydroxymethyl)phosphinic acid (7). Yield $97 \% .{ }^{1} \mathrm{H}$ NMR (200 $\left.\mathrm{MHz}, \mathrm{D}_{2} \mathrm{O}\right) \delta 1.13\left(\mathrm{t}, 3 \mathrm{H}, J=7.1 \mathrm{~Hz}, \mathrm{CH}_{3}\right), 3.00-3.10\left(\mathrm{~m}, 4 \mathrm{H}, \mathrm{CH}_{2} \mathrm{P}+\mathrm{CH}_{2} \mathrm{~N}\right), 3.56(\mathrm{~d}, 2 \mathrm{H}, J=$ $\left.6.7 \mathrm{~Hz}, \mathrm{CH}_{2} \mathrm{OH}\right) .{ }^{13} \mathrm{C} \mathrm{NMR}\left(50 \mathrm{MHz}, \mathrm{D}_{2} \mathrm{O}\right) \delta 10.4\left(\mathrm{CH}_{3}\right), 43.0\left(\mathrm{CH}_{2} \mathrm{CH}_{3}\right), 44.8\left(\mathrm{~d}, J_{P C}=86.0\right.$ $\left.\mathrm{Hz}, \mathrm{CH}_{2} \mathrm{P}\right), 60.0$ (d, $\left.J_{P C}=115.5 \mathrm{~Hz}, \mathrm{CHP}\right) .{ }^{31} \mathrm{P}$ NMR $\left(81 \mathrm{MHz}, \mathrm{D}_{2} \mathrm{O}\right) \delta 28.0$. ES-MS $m / z$ : $(\mathrm{M}-\mathrm{H})$ = 152.1. Anal. Calcd for $\mathrm{C}_{4} \mathrm{H}_{12} \mathrm{NO}_{3} \mathrm{P}$ (153.06): C, 31.38; H, 7.90; N, 9.15\%. Found: $\mathrm{C}, 31.17 ; \mathrm{H}$, 7.99; N, 9.08\%.

$\boldsymbol{N}$-Hexylaminomethane(P-hydroxymethyl)phosphinic acid (9). Yield $98 \% .{ }^{1} \mathrm{H}$ NMR (200 $\left.\mathrm{MHz}, \mathrm{D}_{2} \mathrm{O}\right) \delta 0.65\left(\mathrm{t}, 3 \mathrm{H}, J=7.1 \mathrm{~Hz}, \mathrm{CH}_{3}\right), 1.07-1.25\left(\mathrm{~m}, 6 \mathrm{H}, 3\left(\mathrm{CH}_{2}\right)\right), 1.58\left(\mathrm{bt}, 2 \mathrm{H}, \mathrm{CH}_{2} \mathrm{CH}_{2} \mathrm{~N}\right)$, 2.87-3.04 (m, 4H, $\left.\mathrm{CH}_{2} \mathrm{~N}+\mathrm{CH}_{2} \mathrm{P}\right), 3.51\left(\mathrm{~d}, 2 \mathrm{H}, J=7.5 \mathrm{~Hz}, \mathrm{CH}_{2} \mathrm{OH}\right) .{ }^{13} \mathrm{C} \mathrm{NMR}\left(50 \mathrm{MHz}, \mathrm{D}_{2} \mathrm{O}\right) \delta$ $13.3\left(\mathrm{CH}_{3}\right), 21.7\left(\mathrm{CH}_{2}\right), 25.2\left(\mathrm{CH}_{2}\right), 25.3\left(\mathrm{CH}_{2}\right), 30.5\left(\mathrm{CH}_{2}\right), 44.2\left(\mathrm{~d}, J_{P C}=86.1 \mathrm{~Hz}, \mathrm{CH}_{2} \mathrm{P}\right), 50.0$ $\left(\mathrm{CH}_{2} \mathrm{~N}\right), 60.0\left(\mathrm{~d}, J_{P C}=116.8 \mathrm{~Hz}, \mathrm{CHP}\right) .{ }^{31} \mathrm{P}$ NMR $\left(81 \mathrm{MHz}, \mathrm{D}_{2} \mathrm{O}\right) \delta 27.9$. ES-MS $m / z:(\mathrm{M}-\mathrm{H})=$ 
208.4. Anal. Calcd for $\mathrm{C}_{8} \mathrm{H}_{20} \mathrm{NO}_{3} \mathrm{P}$ (209.12): C, 45.92; H, 9.64; N, 6.69\%. Found: C, 46.19; H, 9.35; N, 6.54\%.

$\mathbf{N}$-Cyclohexylaminomethane(P-hydroxymethyl)phosphinic acid (11). Yield $98 \%$. ${ }^{1} \mathrm{H}$ NMR $\left(200 \mathrm{MHz}, \mathrm{D}_{2} \mathrm{O}\right) \delta$ 1.06-1.35 (m, 6H, $\left.3 \times \mathrm{CH}_{2}\right), 1.40-1.96\left(\mathrm{~m}, 4 \mathrm{H}, 2 \times \mathrm{CH}_{2}\right), 2.95-3.07(\mathrm{~m}, 3 \mathrm{H}$, $\left.\mathrm{CHN}+\mathrm{CH}_{2} \mathrm{P}\right), 3.49\left(\mathrm{~d}, 2 \mathrm{H}, J=6.9 \mathrm{~Hz}, \mathrm{CH}_{2} \mathrm{OH}\right) \cdot{ }^{13} \mathrm{C} \mathrm{NMR}\left(50 \mathrm{MHz}, \mathrm{D}_{2} \mathrm{O}\right) \delta 24.02\left(\mathrm{CH}_{2}\right), 24.5$ $\left(\mathrm{CH}_{2}\right), 28.62\left(\mathrm{CH}_{2}\right), 40.9\left(\mathrm{~d}, J_{P C}=88.6 \mathrm{~Hz}, \mathrm{CH}_{2} \mathrm{P}\right), 59.0\left(\mathrm{~d}, J_{P C}=117.7 \mathrm{~Hz}, \mathrm{CHP}\right), 61.1(\mathrm{CH})$. ${ }^{31} \mathrm{P}$ NMR $\left(81 \mathrm{MHz}, \mathrm{D}_{2} \mathrm{O}\right) \delta$ 28.3. ES-MS $m / z$ : $(\mathrm{M}-\mathrm{H})=206.1$. Anal. Calcd for $\mathrm{C}_{8} \mathrm{H}_{18} \mathrm{NO}_{3} \mathrm{P}$ (207.10): C, 46.37; H, 8.76; N, 6.76\%. Found: C, 46.51; H, 8.59; N, 6.62\%.

Inhibitory activity measurements. Urease was purified from Bacillus pasteurii according to the procedure described previously. ${ }^{16}$ In vitro kinetic measurements of inhibitory potency $\left(\mathrm{IC}_{50}\right.$ and $K_{\mathrm{i}}$ ) were done using standard indophenol method. ${ }^{14}$

\section{Acknowledgements}

This work was supported by the Polish Ministry of Science and Higher Education (grant no IP2010 002270).

\section{References}

1. Hulme, C.; Dietrich, J. Mol. Divers. 2009, 13, 195.

2. Domling, A. Chem. Rev. 2006, 106, 17.

3. Bienayme, H.; Hulme, C.; Oddon, G.; Schmitt, P. Chem. Eur. J. 2000, 6, 3321.

4. Hulme, C.; Gore, V. Curr. Med. Chem. 2003, 10, 51.

5. Moedritzer, K.; Irani, R. R. J. Org. Chem. 1966, 31, 1603.

6. Dziełak, A.: Pawełczak, M.; Mucha, A. Tetrahedron Lett. 2011, 52, 3141.

7. Follmer, C. J. Clin. Pathol. 2010, 63, 424.

8. Kosikowska, P.; Berlicki, Ł. Expert Opin. Ther. Pat. 2011, 21, 945.

9. Burne, R. A.; Chen, Y. M. Microb. Infect. 2000, 2, 533.

10. Amtul, Z.; Atta-ur-Rahman; Siddiqui, R. A.; Choudhary, M. I. Curr. Med. Chem. 2002, 9 , 1323.

11. Pope, A. J.; Toseland, C. D.; Rushant, B.; Richardson, S.; McVey, M.; Hills, J. Dig. Dis. Sci. 1998, 43, 109.

12. Bailie, N. C.; Osborne, C. A.; Leininger, J. R.; Fletcher, T. F.; Johnston, S. D.; Ogburn, P. N.; Griffith D. P. Am. J. Vet. Res. 1986, 47, 2604.

13. Griffith, D.P.; Gleeson, M.J.; Lee, H.; Longuet, R.; Deman, E.; Earle, N. Eur. Urol. 1991, 20, 243. 
14. Vassiliou, S.; Grabowiecka, A.; Kosikowska, P.; Yiotakis, A.; Kafarski, P.; Berlicki, Ł. J. Med. Chem. 2008, 51, 5736.

15. Vassiliou, S.; Kosikowska, P.; Grabowiecka, A.; Yiotakis, A.; Kafarski, P.; Berlicki, Ł. J. Med. Chem. 2010, 53, 5597.

16. Berlicki, Ł.; Bochno, M.; Grabowiecka, A.; Białas, A.; Kosikowska, P.; Kafarski, P. Amino Acids 2011, DOI: 10.1007/s00726-011-0920-4.

17. Albouy, D.; Brun, A.; Munoz, A.; Etemad-Moghadam, G. J. Org. Chem. 1998, 63, 7223.

18. Zefirov, N. S.; Matveeva E. D. ARKIVOC 2008, (i), 1.

19. Abdel-Magid, A. F.; Carson, K. G.; Harris, B. D.; Maryanoff, C. A.; Shah, R. D. J. Org. Chem. 1996, 61, 3849. 